\title{
ON VORTEX TUBE STRETCHING AND INSTABILITIES IN AN INVISCID FLUID
}

\author{
Susan FRIEDLANDER
}

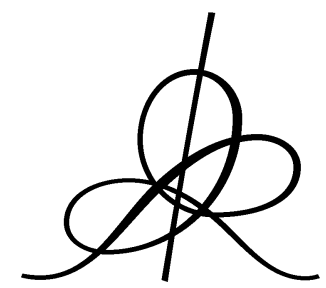

Institut des Hautes Études Scientifiques

35 , route de Chartres

91440 - Bures-sur-Yvette (France)

Août 2000

IHES $/ \mathrm{P} / 00 / 55$ 


\title{
On Vortex Tube Stretching and Instabilities in an Inviscid Fluid
}

\author{
Susan Friedlander \\ Department of Mathematics \\ University of Illinois-Chicago \\ 851 S. Morgan Street \\ Chicago, Illinois 60607 \\ U.S.A.
}

Dedicated to John Heywood on the occasion of his 60 th birthday

\begin{abstract}
We study instabilities that are present in two models that retain some of the dynamics of vortex tube stretching in the motion of a fluid in 3 dimensions. Both models are governed by a 2-dimensional PDE and are hence more tractable than the full 3-dimensional Euler equations. The first model is the so called surface quasi-geostrophic equation. The second model is a class of 3-dimensional flows that are invariant with respect to one spatial coordinate. Both models are constructed in the context of a rapidly rotating fluid. Instabilities due to an effect analogous to vortex tube stretching are detected: these instabilities are in the linearised equations in the first model and in the nonlinear equations in the second model. Such instabilities are absent, or weaker, in strictly 2-dimensional fluid motion.
\end{abstract}




\section{Introduction}

The Euler equations for the motion of an incompressible, inviscid fluid are

$$
\begin{gathered}
\frac{\partial \boldsymbol{q}}{\partial t}+(\boldsymbol{q} \cdot \nabla) \boldsymbol{q}=-\nabla P \\
\nabla \cdot \boldsymbol{q}=0
\end{gathered}
$$

where $\boldsymbol{q}(\boldsymbol{x}, t)$ and $P(\boldsymbol{x}, t)$ are the velocity vector and pressure respectively. We consider these equations in spatial dimensions $D=2$ or 3 . The equation for the vorticity $\boldsymbol{\Omega} \equiv \operatorname{curl} \boldsymbol{q}$ is obtained by taking the curl of (1.1) to give

$$
\frac{\partial \boldsymbol{\Omega}}{\partial t}+(\boldsymbol{q} \cdot \nabla) \boldsymbol{\Omega}=(\boldsymbol{\Omega} \cdot \nabla) \boldsymbol{q} \equiv\left(\frac{\partial \boldsymbol{q}}{\partial \boldsymbol{x}}\right) \boldsymbol{\Omega} .
$$

For motion in 2-D $\Omega=\Omega \widehat{k}$, where $\widehat{k}$ is the unit vector perpendicular to the 2-D plane, hence the R.H.S. of (1.3) is zero and the scalar vorticity satisfies

$$
\left(\frac{\partial}{\partial t}+\boldsymbol{q} \cdot \nabla\right)(\Omega)=0 .
$$

This is the classical result that in 2-D the vorticity is conserved on the trajectories of $\boldsymbol{q}$ and remains bounded for all time. As a consequence of this basic fact fluid motion in 2-D is much less complex than in 3-D. Important mathematical properties of the 2-D Euler equation, such as existence and uniqueness of solutions for appropriate initial and boundary conditions, are rather well understood (see, for example, Kato [17]). The picture is quite different in 3-D where the term $(\boldsymbol{\Omega} \cdot \nabla) \boldsymbol{q}$ is in general non-zero and can, by a mechanism known as vortex tube stretching, generate vorticity. This allows for the development of structures with very complex topology that are closely connected with instabilities and the possible development of singularities. It is an open question in 3 -D whether or not there exists a solution to (1.1)-(1.2) that is initially smooth but develops singularities at some finite time later. Beale et al [4] proved that it is the maximum norm of vorticity that controls the possible breakdown of smooth solutions. Hence the temporal growth of vorticity is a significant (and open) problem for 3-D fluid motion.

Partly because of the seeming intractability of the full 3-D Euler equations a number of authors have considered reduced models which retain to some degree the effects of $(\boldsymbol{\Omega} \cdot \nabla) \boldsymbol{q}$ in the vorticity equation without its full complexity. The primary physical force that is invoked to construct many of these models is rapid rotation (see, for example, [2], [8], [16]). The constraint of rotation limits the freedom of motion of a fluid moving in $3-\mathrm{D}$ and hence leads to approximate 
models that are simpler than the full system. Density stratification also imposes a tendency towards 2-dimensionality on a 3-D flow. Furthermore rotation and stratification are the crucial ingredients in any mathematical model for the ocean or atmosphere and hence such reduced models have practical applications (cf. [19], [18]).

In this paper we consider the effects of a vortex tube stretching type term on the existence of instabilities in two models where although the underlying PDE is 2-dimensional some of the physics of 3-D flow has been retained. The physical rational for both models has as its basis the constraint of rapid rotation.

In Section 2 we investigate the unstable spectrum with respect to growth in the energy of a perturbation of the linearised "surface quasi-geostrophic" (SQG) equation. This is an approximate model that is derived from the equations of motion for a rapidly rotating, density stratified fluid. It is a 2-D equation for an active scalar $\theta$ where $\widehat{k} \times \nabla \theta\left(\equiv \nabla^{\perp} \theta\right)$ plays a role analogous to the vorticity in (1.3). The singular integral that relates $\left(\frac{\partial \boldsymbol{q}}{\partial \boldsymbol{x}}\right)$ to $\boldsymbol{\Omega}$ in 3 -D has as its analogy the singular integral in 2-D that relates $\left(\frac{\partial \boldsymbol{q}}{\partial \boldsymbol{x}}\right)$ and $\nabla^{\perp} \theta$ (see Constantine et al [5]). High frequency asymptotics applied to the Euler equations by Friedlander and Vishik [9] produced a geometric quantity they called a "fluid Lyapunov exponent" whose supremum determines the maximal growth rate of instabilities in the continuous spectrum of the linearised Euler equations. We calculate this exponent for the SQG equation and show that there are instabilities that are not present in strictly 2-D fluid motion. Furthermore if the steady state has a hyperbolic saddle the instabilities are stronger than those for 2-D Euler. These results support our expectation that the vortex tube stretching term in 3-D Euler is inherently destabilising.

In Section 3 we consider a class of exact solutions to the 3-D Euler equation where the motion is independent of one direction (the axis of rotation for a rotating fluid). Yudovich [21], [22] observed that flows of this type give examples of 3-D motion in which there is temporal growth in the vorticity. Viewing such a flow as a perturbation of a steady state, the temporal growth of the vorticity of a perturbation implies nonlinear instability of the steady state in a norm that measures the magnitude of vorticity. We give an explicit expression for the solution of the vorticity equation for " $2^{+}$" dimensional flows. The vorticity behaves as the tangent vector to a 2 -D flow which can be determined explicitly on each trajectory that is not a stagnation point. In general there are initial conditions that lead to growth of the vorticity which becomes exponentially strong on a trajectory that is itself a hyperbolic fixed point. Hence, as in the SQG model, hyperbolicity plus even a limited form of vortex tube stretching produce strong instabilities. It is to be expected that such mechanisms for in- 
stability occur in the full 3-D Euler equations and are an important ingredient in the appearance of turbulence as a consequence of instability to small but finite disturbances that generate vorticity.

\section{Surface Quasi Geostrophic Equation}

In a series of papers Friedlander and Vishik [9], [10], [11] developed and exploited a "geometric optics" type analysis to detect instabilities in the Euler equations, in 2 or 3 dimensions, linearised about a steady state with a smooth velocity $\boldsymbol{U}(\boldsymbol{x})$. The linearised Euler equation for a perturbation velocity $\boldsymbol{v}(\boldsymbol{x}, t)$ is

$$
\begin{gathered}
\frac{\partial \boldsymbol{v}}{\partial t}+(\boldsymbol{U} \cdot \nabla) \boldsymbol{v}+(\boldsymbol{v} \cdot \nabla) \boldsymbol{U}=-\nabla p \\
\nabla \cdot \boldsymbol{v}=0
\end{gathered}
$$

with initial condition $\boldsymbol{v}(\boldsymbol{x}, 0)=\boldsymbol{v}_{0}(\boldsymbol{x}) \in L^{2}$ and $\nabla \cdot \boldsymbol{v}_{0}=0$. We consider free space or periodic boundary conditions. High frequency asymptotics are used to define a geometric quantity $\Lambda$ which is called a fluid Lyapunov exponent. Like the classical Lyapunov exponent which measures exponential growth of a tangent vector to a flow, the fluid Lyapunov exponent measures stretching in fluid motion. The positivity of $\Lambda$ implies instability in the continuous spectrum of the linearised Euler operator acting on the space of square integrable, divergence free vectors. In fact the maximal fluid Lyapunov exponent determines the essential spectral radius, i.e. the maximal growth rate due to the continuous spectrum (Vishik [20]). $\Lambda$ is defined by the following system of O.D.E.:

$$
\begin{aligned}
\frac{d \boldsymbol{x}}{d t} & =\boldsymbol{U}(\boldsymbol{x}) \\
\frac{d \boldsymbol{\xi}}{d t} & =-\left(\frac{\partial \boldsymbol{U}}{\partial \boldsymbol{x}}\right)^{T} \boldsymbol{\xi} \\
\frac{d \boldsymbol{b}}{d t} & =-\left(\frac{\partial \boldsymbol{U}}{\partial \boldsymbol{x}}\right) \boldsymbol{b}+2\left[\left(\frac{\partial \boldsymbol{U}}{\partial \boldsymbol{x}}\right) \boldsymbol{b} \cdot \xi\right] \boldsymbol{\xi} /|\boldsymbol{\xi}|^{2}
\end{aligned}
$$

with $\boldsymbol{x}(0)=\boldsymbol{x}_{0}, \boldsymbol{\xi}(0)=\boldsymbol{\xi}_{0}, \boldsymbol{b}(0)=\boldsymbol{b}_{0}$ and, by definition,

$$
\Lambda \equiv \Lambda(\boldsymbol{U})=\lim _{t \rightarrow \infty} \frac{1}{t} \log \sup _{\substack{\boldsymbol{x}_{0}, \boldsymbol{\xi}_{0}, \boldsymbol{b}_{0} \\\left|\boldsymbol{\xi}_{0}\right|=1, \boldsymbol{b}_{0} \mid=1 \\ \boldsymbol{\xi}_{0} \boldsymbol{b}_{0}=0}}\left|\boldsymbol{b}\left(\boldsymbol{x}_{0}, \boldsymbol{\xi}_{0}, \boldsymbol{b}_{0} ; t\right)\right| .
$$

Here $\frac{d}{d t} \equiv \frac{\partial}{\partial t}+\boldsymbol{U} \cdot \nabla$ denotes the derivative on the trajectories of $\boldsymbol{U}$ and $\left(\frac{\partial \boldsymbol{U}}{\partial \boldsymbol{x}}\right)$ denotes the Jacobian matrix. 
Equation (2.4) is called the co-tangent equation and equation (2.5) the bicharacteristic amplitude equation to the flow $\boldsymbol{U}(\boldsymbol{x})$. Heuristically the equations can be obtained as the leading order equations for perturbations of the form is

$$
\boldsymbol{v}(\boldsymbol{x}, t)=\boldsymbol{b}(\boldsymbol{x}, t) e^{i S(\boldsymbol{x}, t) / \delta}+0(\delta)
$$

where

$$
\boldsymbol{\xi}(\boldsymbol{x}, t)=\nabla S+0(\delta) .
$$

Taking curl of (2.7) gives, to leading order, the perturbation vorticity

$$
\boldsymbol{\omega}=\frac{i}{\delta}(\boldsymbol{b} \times \boldsymbol{\xi}) e^{i S / \delta}+0(1) .
$$

Using (2.4) and (2.5) to calculate the evolution of $\boldsymbol{b} \cdot \boldsymbol{\xi}$ and $\boldsymbol{b} \times \boldsymbol{\xi}$ gives

$$
\frac{d}{d t}(\boldsymbol{b} \cdot \boldsymbol{\xi})=0 .
$$

Since incompressibility requires $\boldsymbol{b}_{0} \cdot \boldsymbol{\xi}_{0}=0$, it follows from (2.10) that

$$
\boldsymbol{b} \cdot \boldsymbol{\xi}=0 \text {. }
$$

Also

$$
\frac{d}{d t}(\boldsymbol{b} \times \boldsymbol{\xi})=-\boldsymbol{b} \times\left(\left(\frac{\partial \boldsymbol{U}}{\partial \boldsymbol{x}}\right)^{T} \boldsymbol{\xi}\right)-\left(\left(\frac{\partial \boldsymbol{U}}{\partial \boldsymbol{x}}\right) \boldsymbol{b}\right) \times \boldsymbol{\xi} .
$$

In 3-dimensions the R.H.S. of (2.12) is, in general, non zero. Identifying $\boldsymbol{b} \times \boldsymbol{\xi}$ with the vorticity (see 2.9), interprets the R.H.S. of (2.12) as the linearisation of the vortex tube stretching term in the $3-\mathrm{D}$ vorticity equation (1.3).

In 2-dimensions the R.H.S. of (2.12) is zero. This follows since $\left(\left(\frac{\partial \boldsymbol{U}}{\partial \boldsymbol{x}}\right) \boldsymbol{\xi}\right.$ $\left.-\left(\frac{\partial \boldsymbol{U}}{\partial \boldsymbol{x}}\right)^{T} \boldsymbol{\xi}\right) \cdot \boldsymbol{\xi}=0$ and $\boldsymbol{b} \cdot \boldsymbol{\xi}=0$, we can write $\left(\frac{\partial \boldsymbol{U}}{\partial \boldsymbol{x}}\right)^{T} \boldsymbol{\xi}=\left(\frac{\partial \boldsymbol{U}}{\partial \boldsymbol{x}}\right) \boldsymbol{\xi}+c(t) \boldsymbol{b}$ for some scalar $c(t)$. Hence from (2.12)

$$
\frac{d}{d t}(\boldsymbol{b} \times \boldsymbol{\xi})=- \text { Trace }\left(\frac{\partial \boldsymbol{U}}{\partial \boldsymbol{x}}\right)(\boldsymbol{b} \times \boldsymbol{\xi})=0
$$

since $\nabla \cdot \boldsymbol{U}=0$. This is a restatement of conservation of vorticity in 2 dimensions for perturbations of the form given by (2.7). From (2.11) and (2.13) it follows that

$$
\frac{d}{d t}(|\boldsymbol{b}||\boldsymbol{\xi}|)=0
$$


hence $|\boldsymbol{b}||\boldsymbol{\xi}|=$ constant. Thus exponential growth of $|\boldsymbol{b}|$, i.e. positivity of $\Lambda$, requires exponential decay of $|\boldsymbol{\xi}|$. Since the flow $\boldsymbol{U}(\boldsymbol{x})$ is volume preserving it follows that in 2 dimensions the fluid Lyapunov exponent and the classical Lyapunov exponent are equal. Hence in 2-dimensions the only nondegenerate flow $\boldsymbol{U}(\boldsymbol{x})$ for which $\Lambda$ is positive, is a flow with a hyperbolic fixed point.

In 3-dimensions we expect the additional stretching mechanisms to produce additional, and possibly stronger, instabilities than those that occur in strictly 2-dimensions. In order to investigate this we will examine not the full 3-dimensional problem but rather a model equation known as the surface quasi geostrophic (SQG) equation. This equation has received considerable attention recently because although it is analytically simpler than the 3-dimensional Euler equation, it retains a number of crucial features that are analogous to those of equations (1.1)-(1.2). This mathematical and physical analogy is described in detail by Constantine et al [5]. The SQG model is a PDE for an active scalar which represents temperature $\theta$ evolving on a 2-dimensional boundary of a rapidly rotating, density stratified, inviscid fluid. It is derived from the full Euler equations in a rotating coordinate system with the addition of a buoyancy term. Under geophysically valid scaling, several approximations are made and the potential vorticity (i.e. the augmented vorticity of a stratified fluid in a rotating frame of reference) is set to zero. A description of the derivation of the SQG equation is given in the books of Pedlosky [10], Salmon [19]. The geophysical relevance of this equation and the ubiquitous generation of secondary instabilities associated with the SQG model is discussed by Held et al [14].

The SQG equation has the following form for a scalar field $\theta(\boldsymbol{x}, t)$ :

$$
\left(\frac{\partial}{\partial t}+\boldsymbol{q}_{H} \cdot \nabla\right) \theta=0
$$

where the 2-dimensional velocity $\boldsymbol{q}_{H}$ and the active scalar $\theta$ are coupled via a stream function $\psi(x, y, t)$, namely

$$
\boldsymbol{q}_{H}=\nabla^{\perp} \psi \equiv\left(-\psi_{y}, \psi_{x}\right)
$$

and

$$
\theta=-(-\Delta)^{1 / 2} \psi
$$

where $(x, y)$ denote cartesian coordinates for a 2-dimensional plane. The nonlocal operator $(-\Delta)^{1 / 2}$ is determined through the 2-dimensional Fourier transform

$$
\psi(x, y, t)=\int e^{2 \pi i \boldsymbol{k} \cdot \boldsymbol{x}} \widehat{\psi}(\boldsymbol{k}, t) d \boldsymbol{k}
$$


by

$$
(-\Delta)^{1 / 2} \psi=\int e^{2 \pi i \boldsymbol{k} \cdot \boldsymbol{x}} 2 \pi|\boldsymbol{k}| \widehat{\psi} d \boldsymbol{k} .
$$

Taking $\nabla^{\perp}$ of $(2.15)$ gives

$$
\left(\frac{\partial}{\partial t}+\boldsymbol{q}_{H} \cdot \nabla\right) \nabla^{\perp} \theta=\left(\frac{\partial \boldsymbol{q}_{H}}{\partial \boldsymbol{x}}\right) \nabla^{\perp} \theta .
$$

It is clear that (2.16) has a superficial resemblence to the 3 -dimensional vorticity equation (1.3) with $\nabla^{\perp} \theta$ playing the role of the vorticity $\boldsymbol{\Omega}$. Constantine et al [5] show that this resemblence is more than superficial when they construct physical, geometric and analytic analogies between (2.20) and (1.3). In particular, the level sets of $\theta$ correspond to vortex lines in (1.3). Both types of curves move with the flow and the nonlocal equation for the evolution of the tangent vectors to the level sets is completely analogous to the equation of vortex stretching. Cordoba [6] explores scenarios for the possible development of singularities in (2.20) as a model for (1.3) and rules out singularities caused by the closing of a hyperbolic saddle.

In this present paper we investigate instabilities in the SQG equation. We apply high frequency asymptotics of the type discussed at the begining of this section to the linearised SQG equation. We note that the geometric construction we described for the fluid Lyapunov exponent applies directly to an augmented system of ODE in the case of a stratified fluid (see [11]). The existence of an exponentially growing vector $\boldsymbol{b}$ that is the amplitude of a perturbation velocity implies the presence of a continuous unstable spectrum. The spectrum of the linearised SQG equation will encompas a subset of possible instabilities of the 3-dimensional stratified Euler equations. Instabilities in the continuous spectrum can be detected by the construction of an exponent $\Lambda$ that is the exponential growth rate of the amplitude of a velocity perturbation governed by the linearisation of (2.15)-(2.17).

Let $\psi_{0}(x, y)$ be the stream function for a steady 2-dimensional flow. The time independent form of $(2.15)-(2.17)$ gives

$$
(\boldsymbol{U} \cdot \nabla) \theta_{0}=0
$$

where

$$
\begin{aligned}
\boldsymbol{U} & =\nabla^{\perp} \psi_{0} \\
\theta_{0} & =-(-\Delta)^{1 / 2} \psi_{0} .
\end{aligned}
$$

We consider the following class of solutions to (2.21)-(2.23), namely functions $\psi_{0}$ that satisfy an elliptic PDE of the form

$$
\theta_{0}=(-\Delta)^{1 / 2} \psi_{0}=g\left(\psi_{0}\right)
$$


hence

$$
\begin{aligned}
\Delta \psi_{0} & =-g^{\prime}\left(\psi_{0}\right) g\left(\psi_{0}\right) \\
& \equiv-f\left(\psi_{0}\right)
\end{aligned}
$$

where $g\left(\psi_{0}\right)$ is a given smooth function of one real variable. Let $\psi_{1}(x, y, t)$ be a small perturbation of the stream function. The linearisation of (2.15) about $\psi_{0}$ gives

$$
\left(\frac{\partial}{\partial t}+\boldsymbol{U} \cdot \nabla\right) \theta_{1}+\nabla^{\perp} \psi_{1} \cdot \nabla \theta_{0}=0
$$

with

$$
\theta_{1}=-(-\Delta)^{1 / 2} \psi_{1}
$$

Let

$$
L \psi_{1} \equiv(\boldsymbol{U} \cdot \nabla)\left((-\Delta)^{1 / 2} \psi_{1}\right)+\nabla^{\perp} \psi_{1} \cdot \nabla \theta_{0} .
$$

This is the operator that is the SQG analogue of the linearised Euler operator in 3 -dimensions. Following the treatment of the linearised Euler operator given in Friedlander and Vishik [9] and Vishik [20] we consider $L$ acting on the space of functions $\psi_{1}$ where $\nabla^{\perp} \psi_{1}$ is square integrable. The positivity of the Lyapunov exponent $\Lambda_{\mathrm{SQG}}$ for the SQG equations detects instabilities in the continuous spectrum of the operator $e^{L t}$. The maximal value of this exponent gives the essential spectral radius for $e^{L t}$.

By analogy with the heuristics for the high frequency asymptotic treatment for the Euler equation we write

$$
\psi_{1}=\beta(x, y, t) e^{i S(x, y, t) / \delta}+0(\delta)
$$

with

$$
\boldsymbol{\xi}=\nabla S .
$$

Hence the leading order contributions to the amplitude of the perturbation velocity, vorticity, and temperature are

$$
\begin{aligned}
\boldsymbol{v} & =\left(\frac{i}{\delta} \beta \widehat{k} \times \boldsymbol{\xi}\right) e^{i S / \delta}+0(1) \\
\boldsymbol{\omega} & =\left(-\frac{\beta}{\delta^{2}}|\xi|^{2} \widehat{k}\right) e^{i S / \delta}+0(1 / \delta) \\
\theta_{1} & =\left(-\frac{i}{\delta} \beta|\boldsymbol{\xi}|\right) e^{i S / \delta}+0(1)
\end{aligned}
$$


where $\widehat{k}$ is the unit vector perpendicular to the $(x, y)$ plane. The SQG analogue of the system of ODE (2.3)-(2.5) are

$$
\begin{aligned}
\frac{d \boldsymbol{x}}{d t} & =\boldsymbol{U}(\boldsymbol{x}) \\
\frac{d \boldsymbol{\xi}}{d t} & =-\left(\frac{\partial \boldsymbol{U}}{\partial \boldsymbol{x}}\right)^{T} \boldsymbol{\xi} \\
\frac{d}{d t}(\beta|\boldsymbol{\xi}|) & =\beta(\widehat{k} \times \boldsymbol{\xi}) \cdot \nabla \theta_{0}
\end{aligned}
$$

with initial conditions $\boldsymbol{x}(0)=\boldsymbol{x}_{0}, \boldsymbol{\xi}(0)=\boldsymbol{\xi}_{0}, \beta(0)=\beta_{0}$. Here $|\beta \boldsymbol{\xi}|$ plays the role of $|\boldsymbol{b}|$. The fluid Lyapunov exponent for the SQG model is

$$
\Lambda_{\mathrm{SQG}}=\lim _{t \rightarrow \infty} \frac{1}{t} \log \sup _{\substack{\beta_{0}, \boldsymbol{x}_{0}, \boldsymbol{\xi}_{0} \\\left|\beta_{0}\right|=1,\left|\boldsymbol{\xi}_{0}\right|=1}}|\beta \boldsymbol{\xi}| .
$$

As we discussed at the begining of this section, in the case of strictly 2dimensional Euler equations the vorticity is constrained so that $\frac{d}{d t}(\boldsymbol{b} \times \boldsymbol{\xi})=0$ and hence $|b||\xi|=$ constant. This severely curtails the circumstances underwhich $|\boldsymbol{b}|$ can grow. The evolution of the vorticity for the SQG equations is not similarly constrained. From (2.34) and (2.35) we obtain

$$
\frac{d}{d t}\left(\beta|\boldsymbol{\xi}|^{2}\right)=\beta|\boldsymbol{\xi}|(\widehat{k} \times \boldsymbol{\xi}) \cdot \nabla \theta_{0}-\beta\left(\frac{\partial \boldsymbol{U}}{\partial \boldsymbol{x}}\right)^{T} \boldsymbol{\xi} \cdot \boldsymbol{\xi} .
$$

In general, the R.H.S. of (2.37) is not zero and mechanisms exist for growth of the vorticity in the SQG model.

Equation (2.35) can be rewritten using the steady state equation (2.24) to give

$$
\frac{d}{d t}(\beta|\boldsymbol{\xi}|)=-\beta g^{\prime}\left(\psi_{0}\right) \boldsymbol{\xi} \cdot \boldsymbol{U} .
$$

From (2.33) and (2.34)

$$
\begin{aligned}
\frac{d}{d t}(\boldsymbol{\xi} \cdot \boldsymbol{U}) & =-\left(\frac{\partial \boldsymbol{U}}{\partial \boldsymbol{x}}\right)^{T} \boldsymbol{\xi} \cdot \boldsymbol{U}+\boldsymbol{\xi} \cdot\left(\frac{\partial \boldsymbol{U}}{\partial \boldsymbol{x}}\right) \boldsymbol{U} \\
& =0 .
\end{aligned}
$$

Hence $\boldsymbol{\xi} \cdot \boldsymbol{U}$ is constant on each trajectory. Clearly $g^{\prime}\left(\psi_{0}\right)$ is also constant on a fixed trajectory since the level curves of $\psi_{0}$ are the trajectories of the flow $\boldsymbol{U}_{0}$. Hence (2.38) gives

$$
\frac{d}{d t}(\beta|\boldsymbol{\xi}|)=\beta C
$$


Since $|\boldsymbol{\xi}| \neq 0$ we obtain

$$
|\beta \boldsymbol{\xi}|=|\beta \boldsymbol{\xi}|_{t=0} e^{\int_{0}^{t} \frac{C}{|\boldsymbol{\xi}(\tau)|} d \tau}
$$

and hence

$$
\Lambda_{\mathrm{SQG}}=\lim _{t \rightarrow \infty} \sup _{\substack{\boldsymbol{x}_{0}, \boldsymbol{\xi}_{0} \\\left|\boldsymbol{\xi}_{0}\right|=1}} \frac{1}{t} \int_{0}^{t} \frac{C}{|\boldsymbol{\xi}(\tau)|} d \tau
$$

where $C=-g^{\prime}\left(\psi_{0}\right)(\boldsymbol{U} \cdot \boldsymbol{\xi})(0)$.

We now investigate the behaviour of the R.H.S. of (2.31) by considering the time dependence of a co-tangent vector $\boldsymbol{\xi}(t)$ on a given trajectory of $\boldsymbol{U}$. On any trajectory which is itself a stagnation point (i.e. $\boldsymbol{U}=0$ ) the value of $\boldsymbol{\xi} \cdot \boldsymbol{U}$ is zero hence the exponent $\Lambda_{\mathrm{SQG}}$ computed on such a trajectory is zero. On any trajectory which is not a stagnation point an explicit formula for $\boldsymbol{\xi}(t)$ was proved in Friedlander et al [13]. The solution to (2.34) for $\boldsymbol{U} \neq 0$ is

$$
\begin{aligned}
\boldsymbol{\xi}(t)= & C_{1} \boldsymbol{U}(\boldsymbol{x}(t)) /|\boldsymbol{U}(\boldsymbol{x}(t))|^{2} \\
+ & {\left[C_{2}-C_{1} \int_{0}^{t} \frac{\left(\left(\frac{\partial \boldsymbol{U}}{\partial \boldsymbol{x}}\right) \boldsymbol{U}+\left(\frac{\partial \boldsymbol{U}}{\partial \boldsymbol{x}}\right)^{T} \boldsymbol{U}\right) \cdot \widehat{k} \times \boldsymbol{U}}{|\boldsymbol{U}|^{4}}(\boldsymbol{x}(\tau)) d \tau\right] } \\
& \widehat{k} \times \boldsymbol{U}(\boldsymbol{x}(t))
\end{aligned}
$$

where

$$
\boldsymbol{\xi}(0)=\frac{C_{1} \boldsymbol{U}\left(\boldsymbol{x}_{0}\right)}{\left|\boldsymbol{U}\left(\boldsymbol{x}_{0}\right)\right|^{2}}+C_{2} \widehat{k} \times \boldsymbol{U}\left(\boldsymbol{x}_{0}\right) .
$$

Since $\boldsymbol{U}(\boldsymbol{x}) \neq 0$ and bounded we get

$$
\left|C_{1}\right| \leq C|\boldsymbol{\xi}(0)|,\left|C_{2}\right| \leq C
$$

for some constant $C$. Hence from (2.43)

$$
|\boldsymbol{\xi}(t)| \lesssim(1+t)|\boldsymbol{\xi}(0)|, t \geq 0 .
$$

Changing $t \rightarrow-t$ we also find

$$
|\boldsymbol{\xi}(t)| \gtrsim \frac{|\boldsymbol{\xi}(0)|}{(1+t)} .
$$

Indeed if we start at point $\boldsymbol{x}(t)$ and apply the previous argument to $\boldsymbol{-} \boldsymbol{U}$ then the equation for $\boldsymbol{\xi}(t)$ has the same solution just run in the reverse direction. 
We claim that the limiting behaviour of $|\boldsymbol{\xi}(t)|$ given by the inequalities (2.45) and (2.46) is achieved on trajectories that form a hyperbolic saddle. Consider the specific example

$$
\psi_{0}=\sin x \sin y .
$$

In the notation of (2.25) this corresponds to

$$
\begin{aligned}
& f\left(\psi_{0}\right)=2 \psi_{0}, \quad g\left(\psi_{0}\right)=\sqrt{2} \psi_{0}, \\
& \boldsymbol{U}=(-\sin x \cos y, \cos x \sin y) .
\end{aligned}
$$

In a neighbourhood of the hyperbolic stagnation point at $(0,0) \psi_{0}$ can be approximated by $\psi_{0} \simeq x y$. Close to $(0,0)$ the trajectories of $\boldsymbol{U}$ are hyperbolae

$$
x=x_{0} e^{-t} \quad y=y_{0} e^{t}
$$

where $|\boldsymbol{x}(0)|=\sqrt{x_{0}^{2}+y_{0}^{2}}$ is chosen to be non-zero but sufficiently small. The co-tangent equation on a hyperbolic trajectory given by (2.49) is

$$
\frac{d \boldsymbol{\xi}}{d t}=-\left(\begin{array}{cc}
-1 & 0 \\
0 & 1
\end{array}\right) \boldsymbol{\xi}
$$

Hence for small $t$ the local behaviour of a co-tangent vector to $\boldsymbol{U}$ near $(0,0)$ is

$$
\boldsymbol{\xi}_{+}=(1,0) e^{t}, \quad \boldsymbol{\xi}_{-}=(0,1) e^{-t}
$$

i.e.

$$
\left|\boldsymbol{\xi}_{+}\right| \simeq(1+t), \quad\left|\boldsymbol{\xi}_{-}\right| \simeq(1+t)^{-1}, \quad t \text { small } .
$$

Thus on such a trajectory the limiting inequalities (2.45) and (2.46) on the growth and decay $\boldsymbol{\xi}$ with time are achieved. On a trajectory of the flow (2.48) that passes close to a hyperbolic point

$$
\left|\boldsymbol{\xi}_{+}(t)\right|=C_{0}(1+t), \quad\left|\boldsymbol{\xi}_{-}(t)\right|=C_{0}(1+t)^{-1}, \quad t>0
$$

where $C_{0}$ depends on $|\boldsymbol{x}(0)|$ and decays away from a hyperbolic point. Choosing $\boldsymbol{\xi}=\boldsymbol{\xi}_{-}$in (2.42) and calculating $g^{\prime}\left(\psi_{0}\right)$ and $\boldsymbol{U} \cdot \boldsymbol{\xi}_{-}$from (2.48) gives an exponent

$$
\Lambda_{\mathrm{SQG}}=\lim _{t \rightarrow \infty} \frac{1}{t} \int_{0}^{t} C_{0} \sqrt{2} y_{0}(1+\tau) d \tau \rightarrow \lim _{t \rightarrow \infty} t \rightarrow \infty .
$$

A similar calculation gives the same result $\Lambda_{\mathrm{SQG}} \rightarrow \infty$ for trajectories corresponding to more general hyperbolic saddles when the stream function has the local form $\psi_{0}=x y-y^{2} \cot \gamma$ (or a locally conformal map of this curve 
in the neighbourhood of the origin). Thus the essential spectral radius of the operator $e^{L t}$, with $L$ defined by (2.27), is infinite when the unperturbed flow has hyperbolic trajectories. This is in contrast to the purely 2-dimensional Euler operator where $\Lambda$ is finite and given by the largest eigenvalue of the matrix $\left(\frac{\partial \boldsymbol{U}}{\partial \boldsymbol{x}}\right)$ at the hyperbolic points. The dynamic and analytic similarities between the SQG model and the 3-dimensional Euler equations catalogued in [5] suggest that the presence of hyperbolic structures in 3-dimensional flows will imply that $\Lambda \rightarrow \infty$. Hence the SQG model suggests that hyperbolicity implies the existence of strong instabilities as measured by growth in the continuous spectrum in $L^{2}$ of the linearised 3-dimensional Euler equation.

In the neighbourhood of an elliptic point for the flow $\boldsymbol{U}$ (e.g. at $x_{0}=\pi / 2$, $y_{0}=\pi / 2$ in the example $\left.\psi_{0}=\sin x \sin y\right)$ there exists a co-tangent vector $\boldsymbol{\xi}$ to an elliptic trajectory of the form $\boldsymbol{\xi}=\boldsymbol{\xi}_{0} e^{ \pm i a t}$. Hence on such a trajectory $|\boldsymbol{\xi}|=\left|\boldsymbol{\xi}_{0}\right|$. The exponent $\Lambda_{\mathrm{SQG}}$ given by $(2.42)$ computed on an elliptic closed trajectory is

$$
\Lambda_{\mathrm{SQG}}=C \equiv g^{\prime}\left(\psi_{0}\right) \boldsymbol{\xi} \cdot \boldsymbol{U} .
$$

Thus the exponent that arises in the SQG model from the presence of an elliptic point is non-zero. Again this is in contrast to the 2-dimensional Euler equations where the exponent $\Lambda$ arising from an elliptic point is zero. These elliptic point instabilities in the SQG equation are analogous to elliptic instabilities in columnular elliptic vortices in the 3-dimensional Euler equation which have been studied using high frequency asymptotics by a number of authors including Bayly et al [3]. The evolution of an elliptical vortex in the context of SQG dynamics with application to geophysics is discussed by Held et al [14].

One class of steady 3-dimensional Euler flows with presumably chaotic streamlines was identified by Arnold [1]. These are so called Beltrami flows whose velocity $\boldsymbol{U}$ is an eigenfunction of curl. A particular periodic example is an " $A B C$ " flow,

$$
\boldsymbol{U}=(A \sin z+C \cos y, B \sin x+A \cos z, C \sin y+B \cos x) .
$$

These flows are non-integrable for nonzero values of the constants $A, B$ and $C$. However the Lagrangian trajectories have been subject to a number of computer investigations, e.g. Henon [15], Dombre et al [7] which indicate that the trajectories possess intricate hyperbolic and elliptic structures. It appears that the trajectories are dense in certain open areas of 3-dimensional phase space. Stagnation points may occur and when they do there is numerical evidence that they are connected by a web of heteroclinic streamlines. Friedlander et al [12] prove (for some ranges of $A, B$ and $C$ ) that there is a positive lower bound for the fluid Lyapunov exponent $\Lambda$ and hence such flows are unstable. The 
analogy between $A B C$ flows with hyperbolic trajectories and the SQG model with a hyperbolic saddle suggests that for $A B C$ flows not only is $\Lambda$ positive but $\Lambda \rightarrow \infty$, implying the existence of strong instabilities.

\section{Nonlinear Instability in " $2^{+}$" Dimensions}

In this section we present another model of fluid motion that incorporates some of the effects of the generation of vorticity that occur in 3-dimensions but without the full complexity of equations (1.1)-(1.3). As was the case for the SQG model, physical motivation comes from rotating fluids. The Euler equations for an inviscid, incompressible fluid written with respect to a coordinate system rotating with constant angular velocity $\bar{\Omega}$ about an axis in the direction of unit vector $k$ are

$$
\begin{gathered}
\varepsilon\left(\frac{\partial}{\partial t}+\boldsymbol{q} \cdot \nabla\right) \boldsymbol{q}+2 \widehat{k} \times \boldsymbol{q}=-\nabla P \\
\nabla \cdot \boldsymbol{q}=0 .
\end{gathered}
$$

The dimensionless parameter $\varepsilon=\bar{U} / \bar{\Omega} L$ where $\bar{U}$ and $L$ are characteristic velocity and length scales. The additional term $2 \widehat{k} \times \boldsymbol{q}$ that occurs in a rotating coordinate system is Coriolis force. The Rossby number $\varepsilon$ measures the relative strengths of inertia and Coriolis force. In many geophysical contexts $\varepsilon \ll 1$. In this case, to first approximation neglecting the inertia term, (3.1), (3.2) reduces to

$$
2 \widehat{k} \times \boldsymbol{q}=-\nabla P, \quad \nabla \cdot \boldsymbol{q}=0
$$

which are called the equations of geostrophic balance. Taking the curl gives the condition

$$
(\widehat{k} \cdot \nabla) \boldsymbol{q}=0
$$

i.e. flows in geostrophic balance are independent of the coordinate $z$ parallel to the axis of rotation. This constraint, known as the Taylor-Proudman theorem, is a fundamental feature of a rapidly rotating fluid. We will examine a particular class of flows that satisfy this constraint and are exact solutions of (3.1)-(3.2) for all values of $\varepsilon$. We refer to these solutions as " $2^{+}$" dimensional flows since the velocity has components in 3 dimensions but is invariant with respect to one coordinate. We will show that, in contrast with strictly

2-dimensional flows, the vorticity of such flows grows, possibly rapidly, with time.

The following expression

$$
\boldsymbol{q}(x, y, t)=\nabla^{\perp} \psi_{0}(x, y)+W(x, y, t) \widehat{k}
$$


is an exact solution to (1.1)-(1.2) for any smooth functions $\psi_{0}(x, y), W(x, y, t)$ provided

$$
\varepsilon\left(\boldsymbol{U}_{H} \cdot \nabla\right) \boldsymbol{U}_{H}-\nabla \psi_{0}=-\nabla P
$$

and

$$
\varepsilon\left(\frac{\partial}{\partial t}+\boldsymbol{U}_{H} \cdot \nabla\right) W=0
$$

where

$$
\boldsymbol{U}_{H}=\nabla^{\perp} \psi_{0} .
$$

One class of solutions to (3.6) is given by streamfunctions $\psi_{0}(x, y)$ that satisfy an elliptic PDE of the form

$$
\nabla^{2} \psi_{0}=-f\left(\psi_{0}\right)
$$

where $f$ is a given smooth function of one real variable. We consider (3.9) subject to suitable boundary conditions, e.g. doubly periodic in $x$ and $y$ or $\psi_{0}=$ constant on a given closed boundary. There is an extensive literature on this problem whose solutions determine steady, 2-dimensional fluid motion. For any specific solution $\psi_{0}(x, y)$ the vertical component $W(x, y, t)$ is a function satisfying (3.7) with a suitable initial condition.

We remark that flows of the form (3.5) cannot satisfy physical boundary conditions with respect to $z$ : e.g. finite energy in an infinite domain or "rigid lid" conditions $W=0$ at $z=0$. In this sense they can only be an approximate model for a physical problem. However they are consistent with an "interior" solution in a rotating bounded domain which is the first order approximation arising from the Navier-Stokes equations for a viscous fluid. When $W$ is suitably scaled with respect to a viscous parameter (the Ekman number) boundary layer asymptotics can be used to approximate rigid boundary conditions.

The vorticity $\boldsymbol{\Omega}(x, y, t)$ corresponding to the flow (3.5) is

$$
\boldsymbol{\Omega} \equiv \operatorname{curl} \boldsymbol{q}=\nabla^{\perp} W+\widehat{k} \Delta \psi_{0} .
$$

The evolution equation for the vorticity becomes

$$
\left(\frac{\partial}{\partial t}+\boldsymbol{U}_{H} \cdot \nabla\right) \nabla^{\perp} W=\left(\frac{\partial \boldsymbol{U}_{H}}{\partial \boldsymbol{x}}\right) \nabla^{\perp} W
$$

with the non-zero R.H.S. of (3.11) being the restriction to $z$-independent flows of the general 3-dimensional "vortex tube" stretching term on the R.H.S. of (1.3). We note that this equation has the same structure as the "vorticity" equation (2.20) that arises from the SQG model. However $W$, unlike $\theta$, is a 
"passive" scalar that is not coupled to $\boldsymbol{U}_{H}$ to produce a nonlinear equation. Rather, for any given $\psi_{0}$ that satisfies (3.9) plus boundary conditions, it follows from (3.11) that the horizontal vorticity $\nabla^{\perp} W$ is a tangent vector to the flow $\nabla^{\perp} \psi_{0}$. Hence the question of possible growth with time of the vorticity is the question of the time dependence of tangent vectors to 2-dimensional steady fluid flows.

For flows $\boldsymbol{U}_{H}$ that have no stagnation points the tangent equation (3.11) can be solved explicitly on each trajectory $\boldsymbol{x}(t)$. We decompose the initial value of $\nabla^{\perp} W \equiv \boldsymbol{\Omega}_{H}$ at $t=0$ as

$$
\boldsymbol{\Omega}_{H}\left(\boldsymbol{x}_{0}\right)=C_{1} \boldsymbol{U}_{H}\left(\boldsymbol{x}_{0}\right)+C_{2} \frac{\widehat{k} \times \boldsymbol{U}_{H}\left(\boldsymbol{x}_{0}\right)}{\left|\widehat{k} \times \boldsymbol{U}_{H}\left(\boldsymbol{x}_{0}\right)\right|^{2}} .
$$

Then

$$
\boldsymbol{\Omega}_{H}(\boldsymbol{x}(t))=\left(C_{1}+C_{2} \alpha(t)\right) \boldsymbol{U}_{H}(\boldsymbol{x}(t))+C_{2} \frac{\widehat{k} \times \boldsymbol{U}_{H}(\boldsymbol{x}(t))}{\left|\widehat{k} \times \boldsymbol{U}_{H}(\boldsymbol{x}(t))\right|^{2}}
$$

where

$$
\alpha(t)=\int_{0}^{t}\left[\left(\left(\frac{\partial \boldsymbol{U}_{H}}{\partial \boldsymbol{x}}\right)+\left(\frac{\partial \boldsymbol{U}_{H}}{\partial \boldsymbol{x}}\right)^{T}\right)\left(\widehat{k} \times \boldsymbol{U}_{H}\right)\right] \cdot \frac{\boldsymbol{U}_{H}}{\left|\boldsymbol{U}_{H}\right|^{4}}(\boldsymbol{x}(\tau)) d \tau .
$$

The proof follows by direct substitution of (3.13)-(3.14) into the tangent equation (3.11) in the same way as the solution to the co-tangent equation (2.34) was obtained in Friedlander et al [13]. Since $\boldsymbol{U}_{H}$ is bounded and non-zero it follows from (3.13)-(3.14) that

$$
\frac{\left|\boldsymbol{\Omega}_{H}\left(\boldsymbol{x}_{0}\right)\right|}{1+t} \lesssim\left|\boldsymbol{\Omega}_{H}(\boldsymbol{x}(t))\right| \lesssim(1+t)\left|\boldsymbol{\Omega}_{H}\left(\boldsymbol{x}_{0}\right)\right| .
$$

For example, in the particular case of rotational flow $\boldsymbol{U}_{H}=\widehat{\theta} r A(r)$ where $(r, \theta)$ are polar coordinates the expression (3.13) can be computed explicitly to give

$$
\boldsymbol{\Omega}_{H}(\boldsymbol{x}(t))=\frac{C_{2}}{r A(r)} \widehat{r}+\left(C_{1}+\frac{C_{2} t A^{\prime}(r)}{r A^{2}(r)}\right) r A(r) \widehat{\theta} .
$$

In this example equation (3.7) implies

$$
W=F(\theta-t A(r))
$$

where $F$ is any smooth function of one variable. On the trajectory of $\boldsymbol{U}_{H}$ starting at a point $\left(r_{0}, \theta_{0}\right)$ the constants $C_{1}$ and $C_{2}$ can be written in terms of 
$F^{\prime}\left(\theta_{0}\right)$ to give

$$
\boldsymbol{\Omega}_{H}(\boldsymbol{x}(t))=\frac{F^{\prime}\left(\theta_{0}\right)}{r_{0}} \widehat{r}+\left(C+t A^{\prime}\left(r_{0}\right) F^{\prime}\left(\theta_{0}\right)\right) \widehat{\theta} .
$$

For flows $\boldsymbol{U}_{H}$ that possess a hyperbolic stagnation point $\boldsymbol{x}_{*}$ exponential stretching takes place on the trajectory that is itself the stagnation point. There exists a positive Lyapunov exponent for $\boldsymbol{U}_{H}$. Let $\lambda$ be the positive eigenvalue of $\left(\frac{\partial \boldsymbol{U}_{H}}{\partial \boldsymbol{x}}\right)_{\boldsymbol{x}_{*}}$. There exists a sequence $t_{j} \rightarrow \infty$ such that $\boldsymbol{x}\left(t_{j}\right) \rightarrow \boldsymbol{x}_{*}$ and a tangent vector $\boldsymbol{\eta}(t)$ such that $\lim _{t \rightarrow \infty} \frac{1}{t} \log |\boldsymbol{\eta}(t)|=\lambda$. Since the horizontal component of vorticity $\boldsymbol{\Omega}_{H}(t)$ satisfies the tangent equation (3.11) there exist initial conditions that permit a 3 -dimensional Euler flow of the form (3.5) whose vorticity grows exponentially as the sequence $t_{j} \rightarrow \infty$.

We have therefore constructed a large class of examples of exact solutions to the 3-dimensional nonlinear Euler equations whose vorticity grows with time and this growth is rapid when the horizontal component of velocity $\boldsymbol{U}_{H}$ has a hyperbolic fixed point.

Yudovich [21], [22], in the context of possible loss of smoothness of solutions to the Euler equation, discusses mechanisms that could lead to temporal growth of the derivatives of the velocity. He observes that the flow whose vorticity is given by (3.18) is just such an example where a derivative, namely the vorticity, grows linearly with time. Yudovich observes that we can draw an interesting conclusion concerning the intrinsic instability of 3-dimensional flows of the type given by (3.5). Consider the steady flow $\boldsymbol{U}_{H}=\nabla^{\perp} \psi(x, y)$ as a flow in 3-D and let $\widehat{k} W(x, y, t)$ satisfying (3.7) be a perturbation of $\boldsymbol{U}_{H}$. We consider the stability of $\boldsymbol{U}_{H}$ where growth is measured in a norm that includes the magnitude of vorticity, e.g.

$$
\left\|\boldsymbol{q}-\boldsymbol{U}_{H}\right\|=\max _{\boldsymbol{x} \in \mathcal{D}}\left|\operatorname{curl}\left(\boldsymbol{q}-\boldsymbol{U}_{H}\right)\right|+\left\|\boldsymbol{q}-\boldsymbol{U}_{H}\right\|_{1}
$$

where \|\|$_{1}$ is some other norm depending only on the magnitude of $\left(\boldsymbol{q}-\boldsymbol{U}_{H}\right)$ and none of its derivatives. We say that $\boldsymbol{U}_{H}$ is nonlinearly (Lyapunov) stable with respect to this norm if for every $\varepsilon>0$ there exists $\delta>0$ such that $\left\|\boldsymbol{q}-\boldsymbol{U}_{H}\right\|_{t=0}<\delta$ implies $\left\|\boldsymbol{q}-\boldsymbol{U}_{H}\right\|<\varepsilon$ for $t \in[0, \infty)$. Otherwise $\boldsymbol{U}_{H}$ is nonlinearly unstable with respect to this norm. When $\left(\boldsymbol{q}-\boldsymbol{U}_{H}\right)=W(x, y, t) \widehat{k}$ we can choose the initial condition so $\|W\|_{t=0}<\delta$ for any $\delta$. However $\|W\|$ grows with time provided only $\boldsymbol{U}_{H}$ has a trajectory on which there exists a growing tangent vector. Thus by this measure of growth almost all flows $\boldsymbol{U}_{H}$ are nonlinearly unstable: rigid body rotation, i.e. $A(r)$ constant, being perhaps the only example that is stable. We note that such temporal growth 
is strongest when the flow $\boldsymbol{U}_{H}$ has a hyperbolic point. As we discussed in Section 2 the existence of a hyperbolic point in the SQG model implies that the maximal growth rate in the continuous spectrum of the linearised operator tends to infinity. This suggests that there is a close (but as yet not understood) connection between the continuous unstable spectrum of the linearised Euler operator and strong temporal growth of vorticity of solutions to the nonlinear 3-dimensional Euler equations.

\section{Acknowledgments}

We thank ETH, Zurich and IHES, France for their kind hospitality during the writing of this paper. We thank Victor Yudovich for many stimulating conversations and the basic idea in Section 2. We thank Cécile Gourgues for the excellent typing of this paper. Friedlander's research is partially supported by NSF grant DMS-9970977.

\section{References}

[1] Arnold, V.I., Sur la topologie des écoulements stationnaires des fluides parfaits. C.R. Acad. Sci. Paris 261 (1965), 17-20.

[2] Babin, A., Mahalov, A., and Nicolaenko, B., Regularity and integrability of 3-D Euler and Navier-Stokes equations for rotating fluids. Asymptot. Anal. 15 (1997), 103-150.

[3] Bayly, B.J., Holm, D.D., and Lifschitz, A., 3-D stability of elliptical vortex columns in external strain flows. Philos. Trans. Roy. Soc. Lond. Ser A 354 $\mathrm{n}^{\circ} 1709$ (1996), 895-926.

[4] Beale, J.T., Kato, T., and Majda, A., Remarks on the breakdown of smooth solutions for the 3-D Euler equations. Commun. Math. Phys. 94 (1984), 61-66.

[5] Constantine, P., Majda, A., and Takak, E., Formation of strong fronts in the 2-D quasi-geostrophic thermal active scalar. Nonlinearity 7 (1994), $1495-1533$.

[6] Cordoba, D., Nonexistence of simple hyperbolic blow up for the quasigeostrophic equation. Annals of Math. 148 (1998), 1-18. 
[7] Dombre, T., Frisch, U., Greene, J.M., Henon, M., Mehr, A., and Soward, A.M., Chaotic streamlines in the $A B C$ flows. J. Fluid. Mech. 167 (1986), 353-391.

[8] Embid, P.F., and Majda, A., Averaging over fast gravity waves for geophysical flows with arbitrary potential vorticity. Comm. P.D.E. 21 (1996), 619-658.

[9] Friedlander, S., and Vishik, M., Dynamo theory, vorticity generation and exponential stretching. Chaos 1 (1991), 198-205.

[10] Friedlander, S., and Vishik, M., Instability criteria for steady flows of a perfect fluid. Chaos 2 (3) (1992), 455-460.

[11] Friedlander, S., and Vishik, M., Instability criteria in fluid dynamics. Proc. workshop on topological fluid mechanics, NATO ASI 128 (1992), 535-549.

[12] Friedlander, S., Gilbert, A., and Vishik, M., Hydrodynamic instability for certain $A B C$ flows. Geophys. Astrophys. Fluid Dyn. 73 (1993), 97-107.

[13] Friedlander, S., Strauss, W., and Vishik, M., Nonlinear instability in an ideal fluid. Ann. Inst. Henri Poincaré, Analyse Nonlinéaire 14 nº 2 (1997), 187-209.

[14] Held, I., Pierrehumbert, R., Garner, S., and Swanson, K., Surface quasigeostrophic dynamics. J. Fluid Mech. 282 (1995), 1-20.

[15] Henon, M., Sur la topologie des lignes de courant dans un cas particulier. C.R. Acad. Sci. Paris 262 (1966), 312.

[16] Julien, K., Knobloch, E., and Werne, J., A new class of equations for rotationally constrained flows. Theoret. Comp. Fluid Dyn. 11 (1998), 251261.

[17] Kato, T., On classical solutions of the 2-D nonstationary Euler equation. Arch. Rational Mech. Anal. 25 (1967), 188-200.

[18] Pedlosky, J., Geophysical Fluid Dynamics. Springer-Verlag, New York, 1987.

[19] Salmon, R., Lectures On Geophysical Fluid Dynamics. Oxford University Press, New York, 1998.

[20] Vishik, M., Spectrum of small oscillations of an ideal fluid and Lyapunov exponents. J. Math. Pures Appl. 75 (1996), 531-557. 
[21] Yudovich, V.I., On loss of smoothness for the Euler equations. To appear in Chaos, 2000.

[22] Yudovich, V.I., Personal communication. 